\title{
Light neutrino masses from gravitational condensation: the Schwinger-Dyson approach
}

\author{
Gabriela Barenboim $^{1}$, Jessica Turner ${ }^{2, \text { a }}$, Ye-Ling Zhou ${ }^{3}$ \\ ${ }^{1}$ Departament de Fisica Teorica and IFIC, Universitat de Valencia, 46100 Burjassot, Spain \\ 2 Department of Physics, Institute for Particle Physics Phenomenology, Durham University, South Road, Durham DH1 3LE, UK \\ ${ }^{3}$ School of Physics and Astronomy, University of Southampton, Southampton SO17 1BJ, UK
}

Received: 25 February 2021 / Accepted: 30 May 2021 / Published online: 10 June 2021

(C) The Author(s) 2021

\begin{abstract}
In this work we demonstrate that non-zero neutrino masses can be generated from gravitational interactions. We solve the Schwinger-Dyson equations to find a nontrivial vacuum thereby determining the neutrino condensate scale and the number of new particle degrees of freedom required for gravitationally induced dynamical chiral symmetry breaking. We show for minimal beyond the Standard Model particle content, the scale of the condensation occurs close to the Planck scale.
\end{abstract}

\section{Introduction}

Neutrinos are unique amongst the Standard Model (SM) fermions in their mass's tininess, the weakness of their interactions and their capacity to be their own anti-particles. Such features suggest neutrinos acquire their mass differently from the quarks and charged leptons. Many such mass models assume neutrinos are Majorana particles and the most prolifically studied are the seesaw mechanisms [1-10]. Typically the masses of the new particles required to complete the lepton-number violating Weinberg operator are larger than the electroweak scale. In addition to tree-level completions of the Weinberg operator, radiative mass models can explain small neutrino masses with $\mathrm{TeV}$-scale new physics [11-14]. Moreover, explanations of light neutrino masses from extradimensions $[15,16]$ and string theory $[17,18]$ provide alternative possibilities (see Ref. [19] for an extensive overview of models of neutrino masses and mixing).

Neutrino masses emerging from gravitational effects were first discussed in [20] where Planck suppressed higherdimensional operators induced neutrino masses. Furthermore, the possibility of neutrino masses emerging from a gravitationally triggered condensate has been studied in vari-

a e-mail: jessica.turner@durham.ac.uk (corresponding author) ous contexts: in [21] it was shown that enhanced gravitational interactions could trigger the formation of a right handed neutrino condensate which induces dynamical symmetry breaking and thereby generates a Majorana mass for the right handed neutrino. From this, the light neutrino masses are generated via the type-I seesaw mechanism. An advantage of such an approach is that the strongly coupled right handed neutrino condensate can drive inflation [22]. A more direct explanation for light neutrino masses was proposed in [23]. In that work it was postulated that gravitational instantons could induce a low-scale $(\sim 100 \mathrm{meV})$ neutrino condensation which can give rise to light neutrino masses [23]; the phenomenology of which has been studied in depth [24].

In this work, we use the Schwinger-Dyson equations to demonstrate that an enhanced gravitational attraction can trigger the formation of an active neutrino condensate which induces dynamical symmetry breaking. We treat gravity as an effective quantum field theory in the spirit of [25]. With minimal assumptions, we show that a non-trivial vacuum can be achieved and find that the phase transition scale is close to the Planck scale. However, new particle degrees of freedom are required to provide finite support to the condensate. Neutrinos remain free particles below the scale of condensation (similarly to [23]) in analogy to the Nambu-JonaLasinio (NJL) model [26] where the constituent fermions are free (unconfined). Furthermore, due to gravity's democratic nature, it will provide a small mass for all fermions.

The work presented in this paper is structured as follows: in Sect. 2 we review the Schwinger-Dyson equations (see Ref. [27] for an in-depth discussion of Schwinger-Dyson methods in QCD and QED) and discuss the leading order diagram which contributes to gravitationally induced neutrino chiral symmetry breaking. We find that chiral symmetry is preserved if the bare graviton propagator is used. Consequently, we apply the dressed graviton propagator, which is discussed in detail in Sect. 3. Further, in this section, we 
introduce the pertinent parameters upon which the neutrino masses depend: the condensate scale $\Lambda$ and two quantities which parametrise the particle content, $A$ and $B$. We elucidate the challenges of finding the chiral breaking vacuum and present two solutions to the Schwinger-Dyson equations in Sects. 5.1 and 5.2 respectively. Finally, we summarise and make concluding remarks in Sect. 6.

\section{The Schwinger-Dyson equation}

The Schwinger-Dyson equations (SDE) are an infinite tower of integral coupled equations which relate the Green functions of a theory to each other. From this set of coupled equations, all observables of the theory can be calculated. We use the SDE as a tool to demonstrate that active neutrinos, which we assume have zero bare mass, can condense via their gravitational interactions and thereby undergo dynamical chiral symmetry breaking. This phenomenon is ultimately non-perturbative, and the SDE provides a method to derive the neutrino gap equation.

The leading order propagator for a massless fermion is simply $S_{F}=i / \not p$. The full propagator will receive selfenergy corrections which modify its form in the following way

$S_{F}^{\prime}(p)=\frac{i}{\not p-\Sigma(p)}=\frac{i}{\alpha\left(p^{2}\right) \not p-\beta\left(p^{2}\right)}$

where $\alpha\left(p^{2}\right)$ and $\beta\left(p^{2}\right)$ are determined by the relevant selfenergy correction, $\Sigma(p)$, and the dynamically induced mass of the fermion is $m_{F}=\beta\left(p^{2}\right) / \alpha\left(p^{2}\right)$. From Eq. (1), we find the propagator consists of two parts: the Dirac odd component, which is the scalar function $\alpha\left(p^{2}\right)$, and the Dirac even part which is parametrised by $\beta\left(p^{2}\right)$. Using the appropriate Dirac trace we find the correlation of these functions with the self-energy correction to be

$\alpha\left(p^{2}\right)=1-\frac{1}{4 p^{2}} \operatorname{tr}(\not p \Sigma(p)), \quad \beta\left(p^{2}\right)=\frac{1}{4} \operatorname{tr}(\Sigma(p))$.

The leading gravitational self-energy correction, as shown in Fig. 1, to the fermion propagator is given by

$-i \Sigma(p)=\int \frac{d^{4} k}{(2 \pi)^{4}} \tau_{1}^{\mu \nu}(p,-k) S_{F}^{\prime}(k) G_{\mu \nu \rho \sigma}^{\prime}(p-k) \tau_{1}^{\rho \sigma}(k,-p)$,

where $\tau_{1}$ is the fermion-fermion-graviton vertex, $S_{F}^{\prime}$ is the modified fermion propagator and $G^{\prime}$ is the dressed graviton propagator. Using the graviton Feynman rules and substitut-

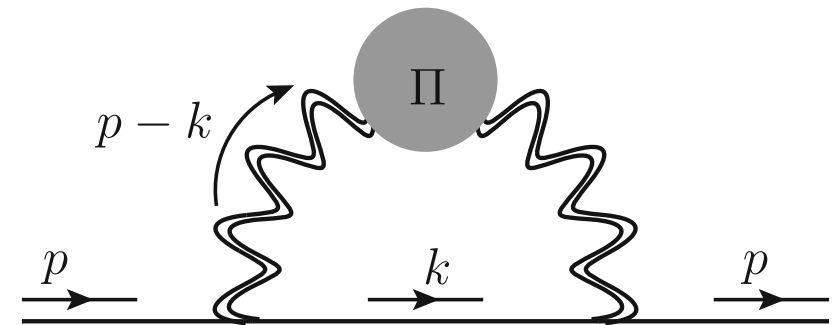

Fig. 1 The self-energy correction to the neutrino propagator. The graviton (represented by the double wavy line) is dressed with the vacuum polarisation (indicated by $\Pi$ ) and external arrows show the momentum flow

ing them into Eq. (3), $\alpha\left(p^{2}\right)$ and $\beta\left(p^{2}\right)$ may be written as

$$
\begin{aligned}
\alpha\left(p^{2}\right)= & 1+\frac{1}{4 p^{2}} \int[d k] \operatorname{tr}\left[\not p \tau_{1}^{\mu \nu}(p,-k) f(k)\right. \\
& \left.\times G_{\mu \nu \rho \sigma}^{\prime}(p-k) \tau_{1}^{\rho \sigma}(k,-p)\right], \\
\beta\left(p^{2}\right)= & -\frac{1}{4} \int[d k] \operatorname{tr}\left[\tau_{1}^{\mu \nu}(p,-k) f(k) G_{\mu \nu \rho \sigma}^{\prime}(p-k)\right. \\
& \left.\tau_{1}^{\rho \sigma}(k,-p)\right] .
\end{aligned}
$$

where

$f(k)=\frac{\alpha\left(k^{2}\right) \not k+\beta\left(k^{2}\right)}{\alpha^{2}\left(k^{2}\right) k^{2}-\beta^{2}\left(k^{2}\right)} \quad$ and $\quad[d k]=\frac{d^{4} k}{(2 \pi)^{4}}$

and we replace the dressed graviton propagator, $G_{\mu \nu \rho \sigma}^{\prime}(p-$ $k$ ), by its tree-level counterpart, $G_{\mu \nu \rho \sigma}(p-k)$, we obtain the leading order contribution of $\alpha\left(p^{2}\right)$

$$
\begin{aligned}
& \alpha\left(p^{2}\right)=1 \\
& -i 2 \pi G \int[d k] f(k) \frac{\left[2(k \cdot p)^{2}+4 k^{2} p^{2}+3 k \cdot p\left(k^{2}+p^{2}\right)\right]}{p^{2}(p-k)^{2}}, \\
& \beta\left(p^{2}\right)=0 .
\end{aligned}
$$

Remarkably, the leading gravitationally induced correction preserves chiral symmetry as $\beta\left(p^{2}\right)$ is exactly zero for all momentum values. The dynamical breaking of chiral symmetry manifests by dressing the graviton propagator with matter fields and the graviton itself at the one-loop order. More specifically, we perform the following replacement

$$
\begin{aligned}
& G_{\mu \nu \rho \sigma}^{\prime}(p-k) \rightarrow G_{\mu \nu \rho \sigma}(p-k) \\
& \quad+G_{\mu \nu \alpha \beta}(p-k) \Pi^{\alpha \beta, \gamma \delta}(p-k) G_{\rho \sigma \gamma \delta}(p-k),
\end{aligned}
$$

where $\Pi^{\alpha \beta, \gamma \delta}(p-k)$ are the vacuum polarisation diagrams as shown in Fig. 2.

\section{Graviton self-energy form factor}

The vacuum polarisation tensor, $\Pi^{\alpha \beta, \gamma \delta}(q)$, which corrects the tree-level graviton propagator is a fourth rank tensor in 

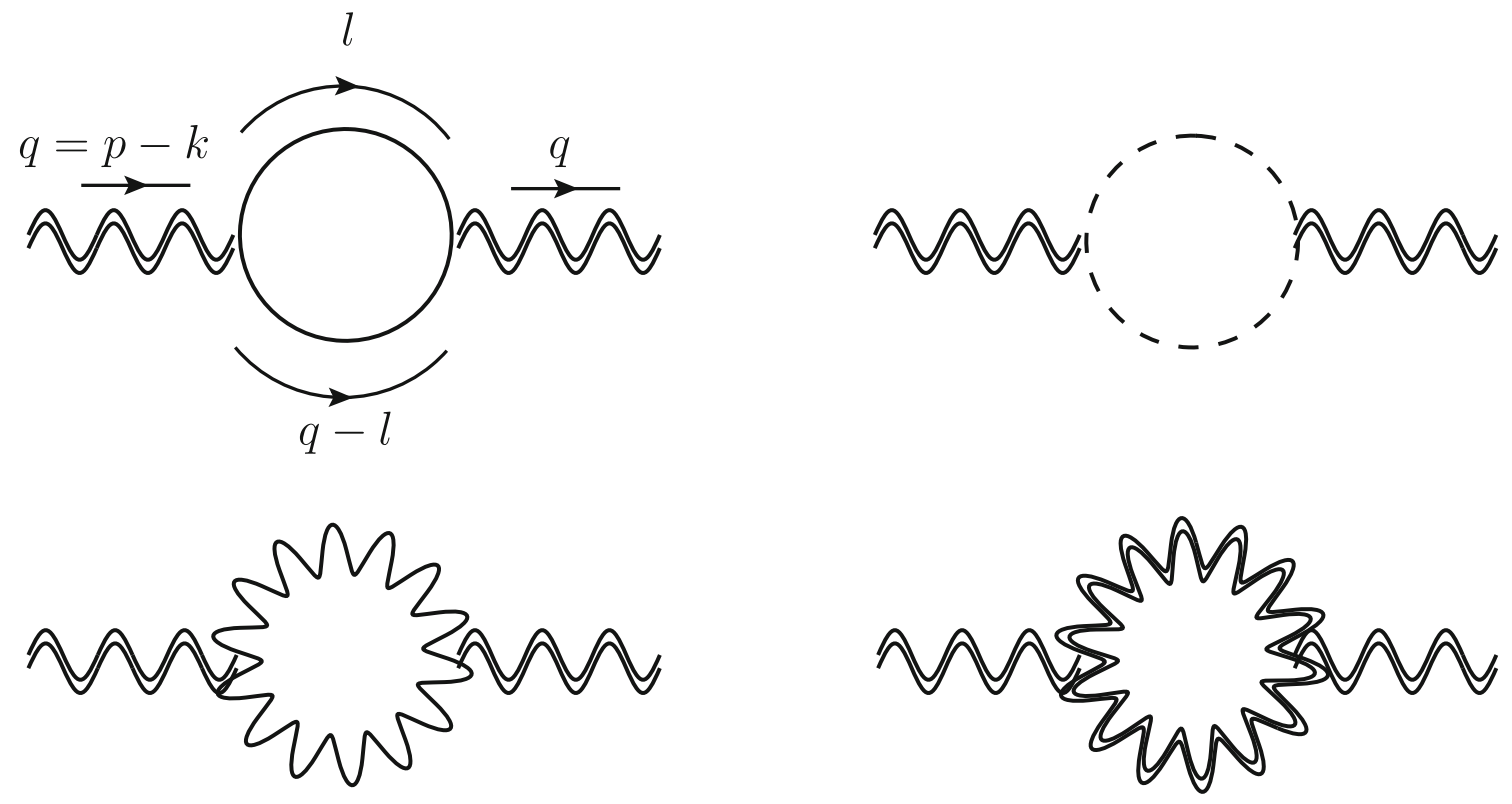

Fig. 2 The set of vacuum polarisations, $\Pi$, which modify the bare graviton propagator is represented by the double wavy lines. The solid line (dotted) indicates a Dirac fermion (minimal scalar) field and the wavy (double wavy) indicates a gauge boson (graviton). The external arrows indicate the momentum flow

$S O(1,3)$. This tensor can be constructed from $\eta^{\alpha \beta}$ and $p^{\alpha}$ and may be written as a linear combination of five independent tensors of rank four:

$$
\begin{aligned}
\Pi^{\alpha \beta, \gamma \delta}(q)= & F_{1}\left(q^{2}\right) q^{\alpha} q^{\beta} q^{\gamma} q^{\delta}+F_{2}\left(q^{2}\right) \eta^{\alpha \beta} \eta^{\gamma \delta} \\
& +F_{3}\left(q^{2}\right)\left(\eta^{\alpha \gamma} \eta^{\beta \delta}+\eta^{\alpha \delta} \eta^{\beta \gamma}\right) \\
& +F_{4}\left(q^{2}\right)\left(q^{\alpha} q^{\beta} \eta^{\gamma \delta}+q^{\gamma} q^{\delta} \eta^{\alpha \beta}\right) \\
& +F_{5}\left(q^{2}\right)\left(q^{\alpha} q^{\gamma} \eta^{\beta \delta}+q^{\alpha} q^{\delta} \eta^{\beta \gamma}\right. \\
& \left.+q^{\beta} q^{\gamma} \eta^{\alpha \delta}+q^{\beta} q^{\delta} \eta^{\alpha \gamma}\right) .
\end{aligned}
$$

The above vacuum polarisation expression is invariant under permutations $\alpha \leftrightarrow \beta, \gamma \leftrightarrow \delta$, as well as $\alpha \beta \leftrightarrow \gamma \delta$ and $F_{i}$ (where $i \in 1,2 \ldots, 5$ ) are a set of form factors. The form factors are not independent of each other as the vacuum polarisation of the graviton must satisfy the Ward identity, $p_{\alpha} \Pi^{\alpha \beta, \gamma \delta}(q)=0$, which leads to three constraints on the five tensors

$$
\begin{aligned}
q^{2} F_{1}+F_{4}+F_{5} & =0, \\
F_{2}+q^{2} F_{4} & =0, \\
F_{3}+q^{2} F_{5} & =0 .
\end{aligned}
$$

As we include up to the one-loop correction to the graviton propagator we parametrise two of these form factors as

$$
\begin{aligned}
& F_{4}\left(q^{2}\right)=a_{1} q^{2} \log \left[\frac{\mu^{2}}{-q^{2}}\right], \\
& F_{5}\left(q^{2}\right)=a_{2} q^{2} \log \left[\frac{\mu^{2}}{-q^{2}}\right],
\end{aligned}
$$

where $\log \left[\mu^{2} /\left(-q^{2}\right)\right]$ comes from the one-loop integration. Using the three constraints of Eq. (10) we derive the following relations

$$
\begin{aligned}
& F_{1}\left(q^{2}\right)=\left(a_{1}+2 a_{2}\right) \log \left[\frac{\mu^{2}}{-q^{2}}\right], \\
& F_{2}\left(q^{2}\right)=-a_{1}\left(q^{2}\right)^{2} \log \left[\frac{\mu^{2}}{-q^{2}}\right], \\
& F_{3}\left(q^{2}\right)=-a_{2}\left(q^{2}\right)^{2} \log \left[\frac{\mu^{2}}{-q^{2}}\right] .
\end{aligned}
$$

Using these constraints, the vacuum polarisations may be parametrised as

$$
\begin{aligned}
& \Pi^{\alpha \beta, \gamma \delta}(q) \\
& =a_{1}\left(q^{\alpha} q^{\beta}-\eta^{\alpha \beta} q^{2}\right)\left(q^{\gamma} q^{\delta}-\eta^{\gamma \delta} q^{2}\right) \log \left[\frac{\mu^{2}}{-q^{2}}\right] \\
& \quad+a_{2}\left[\left(q^{\alpha} q^{\gamma}-\eta^{\alpha \gamma} q^{2}\right)\left(q^{\beta} q^{\delta}-\eta^{\beta \delta} q^{2}\right)\right] \log \left[\frac{\mu^{2}}{-q^{2}}\right] \\
& \quad+a_{2}\left[\left(q^{\alpha} q^{\delta}-\eta^{\alpha \delta} q^{2}\right)\left(q^{\beta} q^{\gamma}-\eta^{\beta \gamma} q^{2}\right)\right] \log \left[\frac{\mu^{2}}{-q^{2}}\right] .
\end{aligned}
$$

Contracting $\Pi^{\alpha \beta, \gamma \delta}\left(q^{2}\right)$ with $\eta_{\alpha \beta} \eta_{\gamma \delta}$ and $\eta_{\alpha \gamma} \eta_{\beta \delta}$ respectively, we obtain

$$
\begin{aligned}
& \Pi_{1} \equiv \eta_{\alpha \beta} \eta_{\gamma \delta} \Pi^{\alpha \beta, \gamma \delta}=\left(9 a_{1}+6 a_{2}\right)\left(q^{2}\right)^{2} \log \left[\frac{\mu^{2}}{-q^{2}}\right], \\
& \Pi_{2} \equiv \eta_{\alpha \gamma} \eta_{\beta \delta} \Pi^{\alpha \beta, \gamma \delta}=\left(3 a_{1}+12 a_{2}\right)\left(q^{2}\right)^{2} \log \left[\frac{\mu^{2}}{-q^{2}}\right] .
\end{aligned}
$$


For any loops contributing to the vacuum polarisation once we calculate the Lorentz-invariants quantities $\Pi_{1}$ and $\Pi_{2}$ from the loop integration, we obtain $a_{1}$ and $a_{2}$, from which we derive the self-energy $\Pi^{\alpha \beta, \gamma \delta}$.

The vacuum polarisations used in this work match those calculated in [21] where the vacuum polarisations to the graviton from the minimal scalar, fermion and gauge bosons were calculated. The vacuum polarisations from the graviton contribution, along with the ghost contribution, were initially calculated in [28] and discussed in [25,29].

Given the graviton Feynman rules for interactions with a minimal scalar (ms), Dirac fermion (df), conformal scalar (cs), gauge boson (gb) and graviton (gr) as provided in Appendix A, we obtain the values of $a_{1}$ and $a_{2}$ as shown in Table 1 . By contracting $\Pi^{\alpha \beta, \gamma \delta}$ with the tree-level propagators $G_{\mu \nu \alpha \beta}(p-k)$ and $G_{\rho \sigma, \gamma \delta}(p-k)$, we determine the dressed graviton propagator $G_{\mu \nu \alpha \beta}^{\prime}(p-k)$ which we substitute into Eq. (4) to find

$$
\begin{aligned}
\beta\left(p^{2}\right)= & i 8 G^{2} \int[d k] f(k)\left[A(k+p)^{2}-B \frac{\left(p^{2}-k^{2}\right)^{2}}{8(p-k)^{2}}\right] \\
& \times \log \left[\frac{\mu^{2}}{-(p-k)^{2}}\right],
\end{aligned}
$$

where $\mu$ is the renormalisation mass which in principle is arbitrary. The degrees of freedom running in the loop diagrams of Fig. 2 are constants given by

$$
\begin{aligned}
& A=\frac{5}{16} \sum_{p}\left(5 a_{1}^{p}+6 a_{2}^{p}\right) N_{p}, \\
& B=\frac{1}{2} \sum_{p}\left(2 a_{1}^{p}+3 a_{2}^{p}\right) N_{p},
\end{aligned}
$$

where $p$ is the index for the particle type (ms, df, gb, cs, gr) and $N^{p}$ is the number of each type of particle. By taking the values of $a_{1}^{p}$ and $a_{2}^{p}$ in Table 1 and fixing the degree of freedom for graviton to be one, we recover the result in Ref. $[21]^{2}$ :

\footnotetext{
1 The above method exploits the transversality of the graviton selfenergy in order to calculate the vacuum polarisations. The "brute force" method can be found in Appendix B.

2 The overall structure of A and B is the same in this work and that of Ref. [21] up to a global factor of eight. This discrepancy stems from an error in Ref. [21]. We note that the SM does not contain any conformal scalars and throughout this work we set $\mathrm{Ncs}=0$. For completeness we provide the expressions for $\mathrm{A}$ and $\mathrm{B}$ and associated coefficients in Table 1.
}

Table 1 Different particles contribution to the graviton self-energy at the one-loop level, $a_{1}$ and $a_{2}$ are coefficients in the graviton form factor $\Pi^{\mu \nu \rho \sigma}$ as shown in Eq. (12)

\begin{tabular}{lcc}
\hline Particle in the loop & $a_{1} / \frac{G}{\pi}$ & $a_{2} / \frac{G}{\pi}$ \\
\hline Minimal scalar & $\frac{1}{40}$ & $\frac{1}{240}$ \\
Dirac fermion & $-\frac{1}{60}$ & $\frac{1}{40}$ \\
Gauge boson & $-\frac{1}{30}$ & $\frac{1}{20}$ \\
Conformal scalar & $-\frac{1}{360}$ & $\frac{1}{240}$ \\
Graviton & $\frac{23}{60}$ & $\frac{7}{40}$ \\
\hline
\end{tabular}

$$
\begin{aligned}
A & =\frac{27 / 2 N_{\mathrm{ms}}+6 N_{\mathrm{df}}+12 N_{\mathrm{gb}}+N_{\mathrm{cs}}+267 N_{\mathrm{gr}}}{288}, \\
B & =\frac{9 N_{\mathrm{ms}}+6 N_{\mathrm{df}}+12 N_{\mathrm{gb}}+N_{\mathrm{cs}}+186 N_{\mathrm{gr}}}{288} .
\end{aligned}
$$

The Standard Model has a large number of degrees of freedom: 12 gauge bosons; 48 chiral fermions and four Higgs scalars. As such the SM values of these parameters are $A=2.61$ and $B=2.27$. However, it is possible there are many more new degrees of freedom at higher energy scales and there are a plethora of theories which consider nonminimal particle content. For example, in the Minimal Supersymmetric Standard Model, these parameters are enlarged such that $A=5.19$ and $B=4.10$. There are other theories with an even richer particle spectrum, for instance the Scalar Democracy as outlined in [30] predicts the existence of 1176 Higgs doublets as a dynamical explanation for the observed fermion mass hierarchy and mixing. In such a theory, $A=223.15$ and $B=149.31$. Moreover, theories which ensure the asymptotic safety of the Standard Model [31] predict similar values of $A$ and $B$ to the work mentioned above. Likewise, a large number of copies $\left(\sim 10^{32}\right)$ of the SM was used to explain the origin and nature of dark matter [32] and correspond to very large $A \sim B \approx 2 \times 10^{32}$.

\section{The kernel structure}

In order to numerically obtain solutions for $\alpha\left(p^{2}\right)$ and $\beta\left(p^{2}\right)$ we rotate the expressions of Eqs. (6) and (14) respectively to Euclidean space. We begin by performing the following replacements, $k^{2}=-k_{E}^{2}, p^{2}=-p_{E}^{2}, d^{4} k=i d^{4} k_{E}$ which modify $\alpha\left(p^{2}\right)$ and $\beta\left(p^{2}\right)$ to take the following form

$$
\begin{aligned}
\alpha\left(p_{E}^{2}\right)= & 1-2 \pi G \int\left[d k_{E}\right] f\left(k_{E}\right) \\
& \times \frac{\left[2\left(k_{E} \cdot p_{E}\right)^{2}+4 k_{E}^{2} p_{E}^{2}+3 k_{E} \cdot p_{E}\left(k_{E}^{2}+p_{E}^{2}\right)\right]}{p_{E}^{2}\left(p_{E}-k_{E}\right)^{2}},
\end{aligned}
$$




$$
\begin{aligned}
\beta\left(p_{E}^{2}\right)= & -8 G^{2} \int\left[d k_{E}\right] f\left(k_{E}\right) \\
& \times\left[A\left(k_{E}+p_{E}\right)^{2}-B \frac{\left(p_{E}^{2}-k_{E}^{2}\right)^{2}}{8\left(p_{E}-k_{E}\right)^{2}}\right] \\
& \times \log \left[\frac{\mu^{2}}{\left(p_{E}-k_{E}\right)^{2}}\right],
\end{aligned}
$$

where $k_{E}^{2}$ and $p_{E}^{2}$ are positive and the Euclidean rotation has changed the relative sign between $\alpha^{2}$ and $\beta^{2}$ in the denominator of the above expression. We note that we have not calculated $\alpha\left(p_{E}^{2}\right)$ using the dressed graviton propagator because its contribution will receive further suppression, by a loop factor, than the leading non-zero undressed contribution. The most straightforward regularisation procedure, which is the one we adopt, is to impose an ultraviolet (UV) cutoff $\Lambda$ on the magnitude of the momentum running in the loop. This UV cutoff is also the condensate scale, above which the chiral symmetry of the neutrino is restored and the condensate dissolves. We expect the UV cutoff to be the same order as $\mu$ given the non-renormalisability of quantum gravity. In QED, a cutoff regularisation scheme is often employed due to its numerical convenience. However, such an approach has its disadvantages as it lacks Lorentz covariance and may lead to ambiguous results. As it has been demonstrated the cutoff regularisation scheme yields qualitatively similar solutions as those derived using off-shell renormalisation [33,34], we proceed with this approach.
We rescale the momentum and $\beta$ such that $p_{E}^{2}=x \Lambda^{2}$, $k_{E}^{2}=y \Lambda^{2}$ and $\beta \rightarrow \beta \Lambda$. In addition, we replace the integral measure, $d^{4} k_{E}$, by the hyper-spherical coordinates, $d^{4} k_{E}=2 \pi \Lambda^{4} y d y \sin ^{2} \theta d \theta$, as well as defining $p_{E} \cdot k_{E}=$ $\sqrt{x y} \Lambda^{2} \cos \theta$ for $\theta \in[0,2 \pi]$ to obtain

$$
\begin{aligned}
& \alpha(x)=1-\frac{G \Lambda^{2}}{(2 \pi)^{2}} \int_{0}^{1} d y \frac{y \alpha(y)}{y \alpha^{2}(x)+\beta^{2}(y)} K(x, y), \\
& \beta(x)=+\frac{8 G^{2} \Lambda^{4}}{(2 \pi)^{3}} \int_{0}^{1} d y \frac{y \beta(y)}{y \alpha^{2}(y)+\beta^{2}(y)} L(x, y) .
\end{aligned}
$$

The variable transformed kernels of Eq. (17) may be written as

$$
\begin{aligned}
K(x, y)= & \frac{1}{x} \int_{0}^{\pi} s_{\theta}^{2} d \theta \frac{2 x y \cos ^{2} \theta+4 x y+3 \sqrt{x y}(x+y) c_{\theta}}{x+y-2 \sqrt{x y} c_{\theta}} \\
L(x, y)= & \int_{0}^{\pi} s_{\theta}^{2} d \theta A\left(x+y+2 \sqrt{x y} c_{\theta}\right) \\
& \times \log \left[x+y-2 \sqrt{x y} c_{\theta}\right] \\
& -B \frac{(x-y)^{2}}{8\left(x+y-2 \sqrt{x y} c_{\theta}\right)} \log \left[x+y-2 \sqrt{x y} c_{\theta}\right]
\end{aligned}
$$

where $s_{\theta}, c_{\theta}$ are $\sin \theta$ and $\cos \theta$. The integrated forms of the above equations shown in Fig. 3 for SM values of $A$ and $B$. In the top left plot of Fig. 3 is the kernel of $\alpha, K(x, y)$. This kernel is large, $K \approx \mathcal{O}(10)$, for $x \sim y$. Because $\alpha$ is non-zero at leading order it is not sensitive to the matter content and is therefore independent of parameters $A$ and $B$. Naturally, this
Fig. 3 For the SM particle content we display the kernels $K(x, y)$ and $L(x, y)=$ $A L_{A}(x, y)+B L_{B}(x, y)$ for $x \in[0,1]$ and $y \in[0,1]$
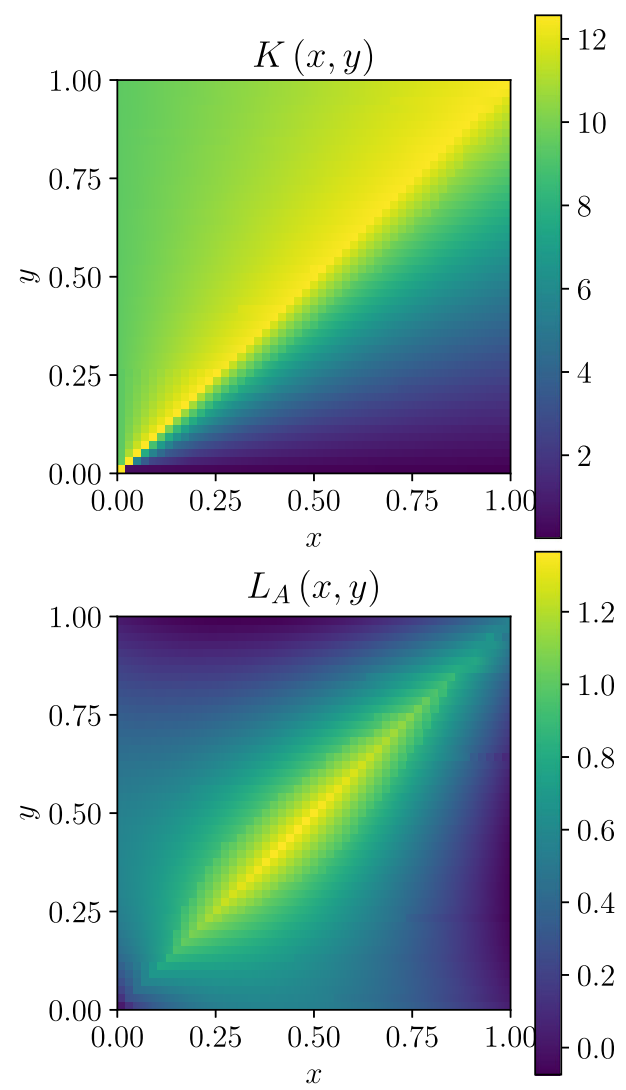

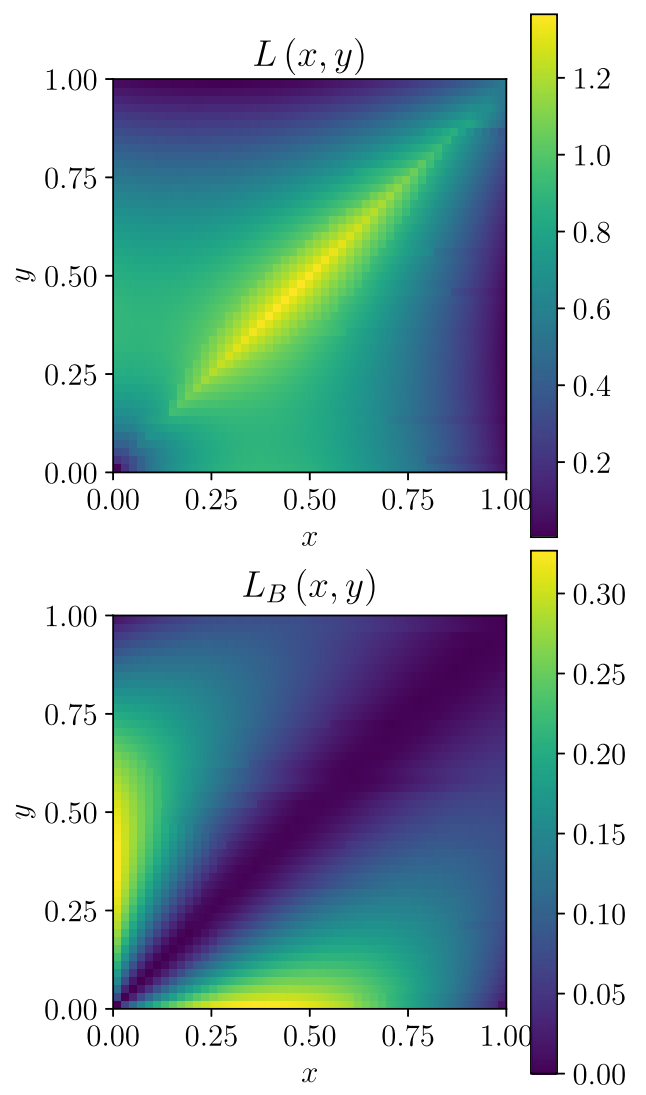


is not the case for the kernel of $\beta, L(x, y)$, which is calculated using the dressed graviton propagator. For the same reasoning, the coefficient of the integrals of $\alpha$ and $\beta$ (given by $G \Lambda^{2}$ and $\left(G \Lambda^{2}\right)^{2}$ respectively) enter with different powers of the UV cutoff. The kernel $L(x, y)$ is split into two components, $L_{A}(x, y)$ and $L_{B}(x, y)$, which are premultiplied by $A$ and $B$ respectively:

$L(x, y)=A L_{A}(x, y)+B L_{B}(x, y)$.

$L_{A}(x, y)$ and $L_{B}(x, y)$ are shown in the bottom left and right plots of Fig. 3. We observe that $L_{A}(x, y)$ is larger by a factor of a few than $L_{B}(x, y)$ for the majority of the $x-y$ region and hence the combined kernel $L(x, y)$ is dominated by $L_{A}(x, y)$. For $x \geqslant y$, the kernels have the simplified form:

$$
\begin{aligned}
K(x, y) & =\frac{\pi}{x} \frac{y}{x}(3 x+y), \\
L_{A}(x, y) & =\frac{\pi}{12}\left[\frac{5 y^{2}-3 x y}{x}-6(x+y) \log x\right], \\
L_{B}(x, y) & =\frac{\pi}{8} \frac{(x-y)^{2}}{x y}[y-x \log x+(x-y) \log (x-y)] .
\end{aligned}
$$

From Eq. (19), we observe the $x K(x, y)$ and $L_{A, B}(x, y)$ are symmetric functions of $x$ and $y$. Therefore, we perform the convenient replacement $x \rightarrow \frac{(x+y)+|x-y|}{2}$ and $y \rightarrow$ $\frac{(x+y)-|x-y|}{2}$ into expressions of $x K(x, y)$ and $L_{A, B}(x, y)$ to obtain

$$
\begin{aligned}
K(x, y) & =\frac{\pi}{x} \frac{(x+y)^{3}-\left[(x+y)^{2}+2 x y\right]|x-y|}{2 x y} \\
L_{A}(x, y) & =\frac{\pi}{12}\left\{\frac{5\left(x^{2}+y^{2}\right)-5(x+y)|x-y|-6 x y}{(x+y)+|x-y|}\right. \\
& \left.-6(x+y) \log \left[\frac{(x+y)+|x-y|}{2}\right]\right\} \\
L_{B}(x, y) & =\frac{\pi}{8} \frac{(x-y)^{2}}{x y}\left\{\frac{(x+y)-|x-y|}{2}\right. \\
& -\frac{(x+y)+|x-y|}{2} \log \left[\frac{(x+y)+|x-y|}{2}\right] \\
& +|x-y| \log (|x-y|)\},
\end{aligned}
$$

which are expression for the kernels of $\alpha$ and $\beta$ respectively if $x>y$ or $x<y$. The analytic manipulation from Eqs. (21) to (22) is applied to make the kernels more amenable for numerical integration.

The non-trivial momentum structure of the $L(x, y)$ kernel may be most easily understood in the language of BCS theory [35] which describes the pairing of fermions. A Cooper pair can form between two fermions of opposite momenta and spin. In such a configuration, the system's energy is minimised, and the fermions combine to give a spin-singlet (or possibly triplet), which leads to an attractive interaction between the fermionic pair. In addition to the spin component, there is also an orbital angular momentum component, $l$, which takes integer values; in the case of an $s$-wave interaction, $l=0$. However, the orbital components can also have other non-trivial integer values, $l=1(p), l=2(d)$ which characterise the pairing. As observed from the kernel structure shown in Fig. 3, $L(x, y)=0$ for $x=y=0$ which corresponds to a $d$-wave interaction. This feature arises due to the spin-2 nature of the graviton.

\section{Numerical solutions to the Schwinger-Dyson equation}

It was postulated in [23] that active neutrinos could condense via gravitational instantons and consequently acquire a mass below energies of $\sim 100 \mathrm{meV}$. In this work, we apply the calculational techniques used to condense right-handed neutrinos from their gravitational interactions [21] to light, active neutrinos.

The practical challenge of this task comes from the great separation in the relevant energy scales. The gravitational coupling is parametrised by $\kappa=\sqrt{32 \pi G} \approx 10^{-18} \mathrm{GeV}^{-1}$. From the SDE we find the coefficients of both $\alpha$ and $\beta$ are proportionate to $G \Lambda^{2}$ and $\left(G \Lambda^{2}\right)^{2}$, respectively. To recover a non-trivial vacuum, the cutoff scale $\Lambda$ cannot be far from the Planck scale $\left(M_{\mathrm{pl}} \approx 10^{19} \mathrm{GeV}\right)$ unless the particle content is enormous. This point will become more apparent in Sect. 5.1. On the other end of the energy scale are the tiny neutrino masses, $m_{v} \sim 10^{-10} \mathrm{GeV}^{3}$ We can contrast this with the relevant scales in $\mathrm{QCD}$, where $\Lambda_{\mathrm{QCD}} \approx 1 \mathrm{GeV}$ and the light quark masses, $m_{u / d} \approx \mathcal{O}(1) \mathrm{MeV}$. We find the separation in scales to be $m_{u / d} / \Lambda_{\mathrm{QCD}} \approx 10^{-3}$. However, in our case of interest, we require a non-zero but very small ratio of scales, $m_{v} / \Lambda_{G} \approx 10^{-29}$. This presents a unique numerical challenge in finding the non-trivial vacuum compared with other gauge theories where the SDE techniques are applied. We present two possible solutions below which demonstrate gravitationally induced chiral symmetry breaking.

\subsection{Extrapolation}

We implement an iterative, numerical method of solving the SDE with a cutoff regularisation a similar method as outlined in [37]. The limitations such an approach are discussed in [38]. We define $\alpha^{(i+1)}$ and $\beta^{(i+1)}$ (for $i=0,1,2,3, \ldots$ ) to be

$$
\begin{aligned}
& \alpha^{(i+1)}(x)=1-\frac{G \Lambda^{2}}{(2 \pi)^{2}} \int_{0}^{1} d y \frac{y \alpha^{(i)}(y)}{y \alpha^{(i)^{2}}(x)+\beta^{(i)^{2}}(y)} K(x, y), \\
& \beta^{(i+1)}(x)=\frac{8\left(G \Lambda^{2}\right)^{2}}{(2 \pi)^{3}} \int_{0}^{1} d y \frac{y \beta^{(i)}(y)}{y \alpha^{(i)^{2}}(y)+\beta^{(i)^{2}}(y)} L(x, y) .
\end{aligned}
$$

\footnotetext{
${ }^{3}$ It was very recently confirmed by the terrestrial experiment KATRIN that the effective neutrino mass measured using beta decay is less than $1.1 \mathrm{eV}(90 \%$ CL) [36].
} 
We choose two trial functions as initial values for the iterative calculation

$\alpha^{(0)}(x)=c_{1}, \quad \beta^{(0)}(x)=c_{2}$,

where $c_{1}$ and $c_{2}$ are constants. As expected, the solutions of $\alpha$ and $\beta$ do not exhibit sensitivity to the value of the trial functions. Also, we require a definition of convergence which we parametrise by the tolerance

tolerance $\equiv \frac{\beta^{(i+1)}(x)}{\beta^{(i)}(x)}-1$.

The procedure for solving Eq. (23) is as follows:

1. Choose a value of $G \Lambda^{2}, A, B$, tolerance and trial function values.

2. Subdivide the $x$-interval $\left[x_{\mathrm{IR}}, 1\right]$ into $n$ bins, where $x_{\mathrm{IR}}$ is infrared boundary of the theory.

3. Iteratively solve Eq. (23) for each bin.

4. For each bin we calculate the tolerance and summate this measure over all bins.

5. Require the solution to be stable as the tolerance is reduced.

To ensure the solution is independent of the number of bins, we normalise the tolerance by the total number of bins. Additionally, we test the solution does not vary for differing values of the tolerance. As the region of interest is in far-infrared, as represented by $x_{\mathrm{IR}}$, to probe the sub-electroweak energy scales requires $x \approx 10^{-34}$. Moreover, as the mass of the neutrino is defined as

$m_{v}=\frac{\beta(0)}{\alpha(0)} \Lambda$,

requires $\beta(0) \approx 10^{-29}$ for $\Lambda \approx M_{\mathrm{pl}}$. Achieving this level of precision in the numerical integration and the endpoint is challenging, and consequently, we solve the iterative SDE in regions where we have numerical control and then extrapolate in the combination $G \Lambda^{2} A$.

In summary, fix $G \Lambda^{2}=1$ and vary $A$ and $B$ (which parametrise the matter content) and find the non-trivial minima which gives rise to a non-zero stable value of $\beta\left(x_{\mathrm{IR}}=\right.$ $10^{-10}$ ). We then extrapolate this function to $x_{\mathrm{IR}}=0$ for $\beta$ to $\beta(x=0)$. We repeat this procedure for several values $A$ and $B$ (for a fixed $G \Lambda^{2}$ ) and calculate the solutions numerically. We fit a polynomial to these points as represented by the red line and blue dots in Fig. 4 respectively. The functional form of this polynomial is

$$
\begin{aligned}
\beta^{2}(0)= & -0.0792286+5.28 \times 10^{-4}\left(G \Lambda^{2} A\right)^{\frac{3}{2}} \\
& -1.14 \times 10^{-5}\left(G \Lambda^{2} A\right)^{2}
\end{aligned}
$$

This is the lowest order polynomial that provides a good fit to the numerically calculated points. To recover neutrino masses of the correct order of magnitude requires $\beta(0) \approx 10^{-29}$ with

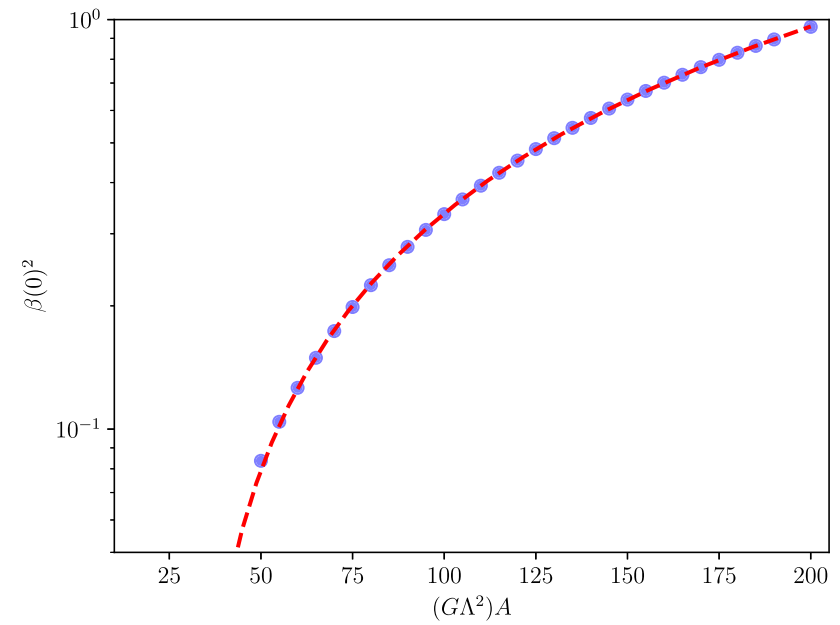

Fig. $4 \beta^{2}(0)$ as a function of $\left(G \Lambda^{2}\right) A$ where $A$ and $B$. We take $A=B$ with $G \Lambda^{2}=1$ and vary the values of $A$. The blue dots are the solutions to the SDE and the red dotted line is the fitted polynomial

$G \Lambda^{2} \approx 1$ implies $A \gtrsim 30$. Therefore new particle content is required to support the condensate and the lower the condensation scale the larger the particle content required for chiral symmetry breaking to occur. As instantons are a modification to the gauge boson propagator, the inclusion of such effects are unlikely to lower the scale substantially. We note that there is a large amount of fine-tuning required to reproduce light neutrinos masses: we must tune the quantity $G \Lambda^{2} A$ such that the solution is very close to but not equal to the chiral preserving solution, $\beta(0)=0$. This tuning is not surprising: in the scenario of minimal new particle content, the neutrino mass is proportionate to $\Lambda$, the only dimensionful parameter of Eq. (26). A non-trivial vacuum requires $G \Lambda^{2} \approx 1$ otherwise the iterative solution of Eq. (23) evolves to the trivial vacuum. Because $G \approx 1 / M_{\mathrm{pl}}^{2}$ this implies $\Lambda \approx M_{\mathrm{pl}}$. Therefore to recover sub-eV masses of neutrinos requires $\beta(0)$ to be very small. It is important to note that this discussion applies only to a minimal number of new degrees of freedom and the conventional scale of quantum gravity. However, as we will further elucidate in Sect. 6, further new physics may ameliorate this fine-tuning.

\subsection{Consistency of $\beta(x)$ with the Kernel structure}

In this method, we exploit the structure of the kernel of $\beta$ to demonstrate the existence of a non-trivial vacuum. Once the true vacuum is found, any value of the infrared mass can be recovered from parameter tuning.

We remind the reader $x$ is the neutrino's ingoing momentum normalised by the UV cutoff and $y$ is the cutoff normalised loop momentum. From Fig. 5, we show the kernel of $\beta, L(x, y)$, for all values of $y$ and $x \in\left[10^{-15}, 10^{-10}\right]$ which 


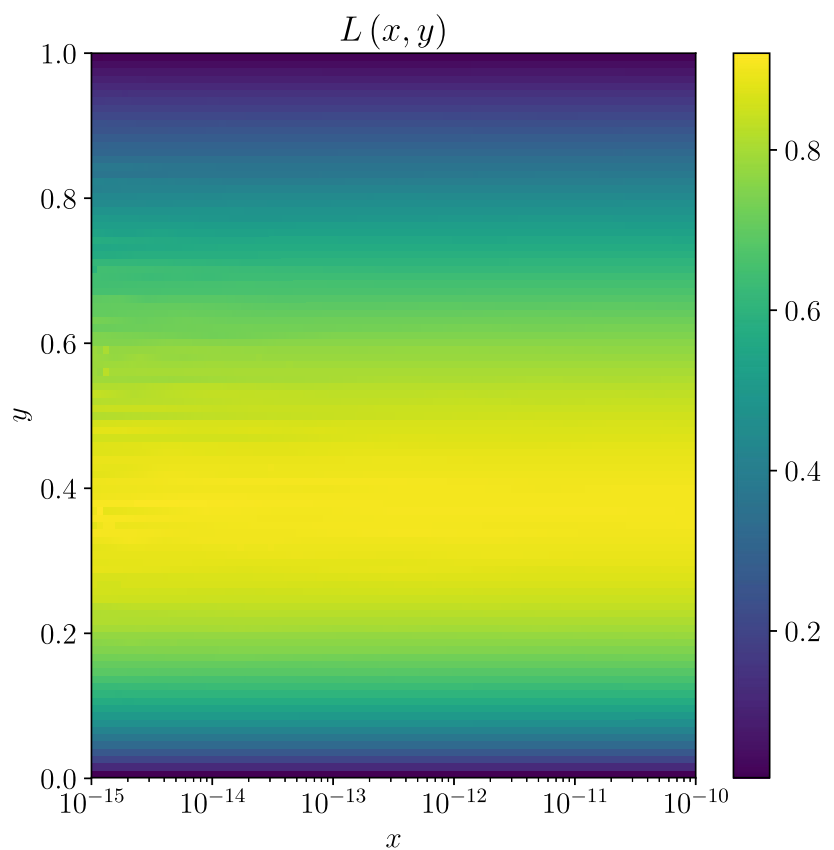

Fig. 5 For the SM particle content we display the kernels $L(x, y)=$ $A L_{A}(x, y)+B L_{B}(x, y)$ for $x \in\left[10^{-15}, 10^{-10}\right]$ and $y \in[0,1]$

demonstrates the far-infrared behaviour of the kernel. ${ }^{4}$ From the kernel structure, we postulate that $\beta(x)$ is insensitive to $x$ until close to the ultraviolet cutoff. As the only $x$ dependence can come from the kernel because $y$ is integrated over, taking the form of $\beta$ to constant in $x$ is beyond a sufficiently good approximation. The same is true for the kernel of $\alpha$. From these considerations, the crudest approximation of $\beta(x)$ is a step function of constant magnitude, $a$, which can be written as

$\beta(x)=\frac{8\left(G \Lambda^{2}\right)^{2}}{(2 \pi)^{3}} \int_{0}^{1} \frac{a y d y}{a^{2}+y} L_{A}(x, y)$,

where the quenched limit has been applied, $\alpha \approx 1$, and the definition of $a$ contains information about the particle content and the overall scale of $\beta$. Since $A>B$ and $L_{A}(x, y)>$ $L_{B}(x, y)$ we have ignored the sub-leading contribution of $B L_{B}(x, y)$. We solve Eq. (28) for a fixed value of $G \Lambda^{2}$ and $a$. For $a=10^{-5}$ and $G \Lambda^{2}=1$ we find the solution to be $\beta(0) \approx$ $4 \times 10^{-6}$ as shown in Fig. 6 . With the appropriate tuning of $G \Lambda^{2}$ and $a$ any non-zero value of $\beta(0)$, and therefore the infrared mass of the neutrino, can be recovered. From Fig. 6, we observe there is some non-trivial $x$ dependence in $\beta(x)$. However, this only occurs for $x \approx 0.4$ which is in the far ultraviolet region of the theory. As we are only interested in the infrared mass and do not make any statements regarding the deep UV physics, this feature does not impact the final result. This approach checks for the self-consistency of the

\footnotetext{
$\overline{4}$ We note that for smaller values of $x\left(x \ll 10^{-15}\right)$ this flat behaviour in $x$ does not change.
}

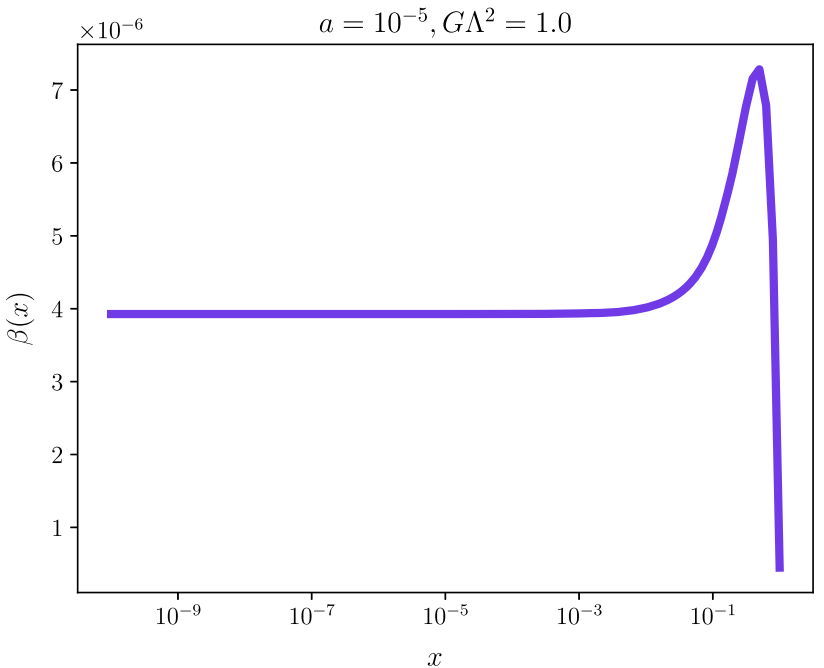

Fig. $6 \beta(x)$ as a function of $x$ from solving Eq. (28) with $a=10^{-5}$ and $G \Lambda^{2}=1$

postulated form of $\beta(x)$ with the kernel structure and agrees with the first method discussed in Sect. 5.1.

\section{Discussion}

In Sects. 5.1 and 5.2 we demonstrated a common neutrino mass scale can be generated through iteratively solving the SDE and also making an informed Ansatz for the form of $\beta(x)$. From the first method, we found there are two factors which support a gravitationally induced neutrino condensate: the scale of the condensate, $\Lambda$, and the particle content as parametrised by $A$ and $B$. These two factors compensate for each other: if the condensation scale is lowered, the particle content must be increased and vice versa. The minimal particle content required for $\Lambda \approx M_{\mathrm{pl}}$ is still larger than the SM; however, this conclusion follows from assuming a conventional Planck scale, $M_{\mathrm{pl}} \approx 10^{19} \mathrm{GeV}$.

An alternative possibility comes from extra compact dimensions $[39,40]$. Given $n$ extra dimensions, with length scale $R$, the number of degrees of freedom for each bulk field $N_{p}$ is proportional to the number of Kaluza-Klein (KK) excitations, and the latter is determined to the cutoff scale, i.e., $N_{p} \sim(\Lambda R)^{n}$ [39]. In this framework, the cutoff scale, $\Lambda$, should be lower than the true Planck scale $M_{*}$ in $4+n$ dimensions, which is correlated with the effective Planck scale $M_{\mathrm{pl}}$ in the four dimensions. For example, in the ADD model [39], $M_{\mathrm{pl}}^{2}=M_{*}^{n+2} R^{n}, N_{p}$ can maximally reach the order $\left(M_{*} / M_{\mathrm{pl}}\right)^{2}$. The true Planck $M_{*}$ is much lower than the effective scale $M_{\mathrm{pl}}$. By assuming the cutoff scale to be just below $M_{*}$, e.g., $\Lambda=0.9 M_{*}$, we obtain $G \Lambda^{2} A \sim \Lambda^{2} N_{p} / M_{\mathrm{pl}}^{2} \sim \mathcal{O}(1)$, which guarantees the model staying in the non-perturbative regime. In this 
regime, the ratio between neutrino mass and the scale is modified into $m_{v} / \Lambda \sim m_{v} / M_{*}$. Furthermore, by taking $n=3$ and $R \sim 10^{-9} \mathrm{~m}$, which is sufficient to evade experimental constraints [41], $M_{*}$ is lowered to the $\mathrm{TeV}$ scale and $m_{v} / \Lambda \sim 10^{-14}$.

In contrast with the vast majority of neutrino mass generation mechanisms, the effect of gravitational condensation is insensitive to the Dirac or Majorana nature of the neutrino. As gravity is universal and does not discriminates between particle species, if a small mass is induced for neutrinos, a small mass will be induced for the other SM fermions at the scale $\Lambda$. Although this scale may be significantly below the Planck scale, a great deal of new matter is required. We note that electroweak symmetry will not be induced as the effect of tadpoling the Higgs is of order of the mass contribution to the neutrinos, which is small. The connection between the gravity induced mass gap for the neutrinos and black holes, which are non-perturbative solutions in general relativity, may be recovered as poles in the resummed graviton propagator could be interpreted as black hole precursors [42,43].

In summary, we have shown that neutrinos can condense via gravitational interactions and undergo chiral symmetry breaking. To do so, we treat gravity as an effective quantum field theory and solve the Schwinger-Dyson equations to find a non-trivial vacuum. The true vacuum is recovered in two ways: the first through iteratively solving the SDE and the second from making an Ansatz for the kernel of $\beta$. In the minimal setup, the scale of the condensation is found to be close to the Planck scale and new degrees of freedom beyond the Standard Model particle content are required. Interestingly, new physics is required to explain neutrino mass scale in this framework: the Standard Model in addition to gravity is insufficient to explain neutrino masses. An important point to note is that this calculation demonstrates a common neutrino mass scale may be gravitationally induced; however, to reproduce oscillation data, a further mechanism is required to break the mass degeneracy. We agree with the conclusions of [23], where a common mass scale is recovered. In that work, the neutrinos' mass splittings are induced from the effective potential of the Goldstone bosons, which is a nongravitational effect. The Goldstone bosons associated with the former mechanism can be relatively light $(\sim \mathrm{MeV})$ and there is interesting associated phenomenology [24]. However, in this work, if the condensate scale is high (and not lowered due to extra-dimensions or new particle content), the mass of the Goldstone bosons would be large and no such low-scale phenomenology would not be observed. On the other hand, if the scale was lowered due to extra-dimensions or new particle content, similar phenomenology to that discussed in [24] would be observed. Furthermore, lowering the condensate scale would have interesting cosmological consequences: as the neutrino mass changes on cosmological timescales, its mass can be reconstructed as a function of redshift [44].

We find that with minimal new particle content, the condensation scale is high and close to the Planck scale. The contribution of the condensate to neutrino masses is small and equal for the three neutrino masses. The condensate scale can be lowered if there is a significant increase in the number of degrees of freedom and in such a case, the contribution of the condensate to the three neutrino masses would still be small and equal. This compensatory effect in supporting the condensate does not fully overcome the large fine-tuning required to recover tiny neutrino masses. However, if the true Planck scale is lowered than expected, due to extra compact dimensions, such tuning could be somewhat reduced.

Acknowledgements We are grateful to Craig Roberts for generously sharing his knowledge of the Schwinger Dyson equations and nonperturbative physics with us. It is a pleasure to thank Simon Badger, Bill Bardeen, Nikita Blinov and Pedro Machado for useful discussions on various aspects of this work. We thank Niels BjerrumBohr and Apostolos Pilaftsis for their correspondence regarding the graviton-fermion-fermion Feynman rules. We are grateful to Joannis Papavassiliou for useful discussion on non-perturbative techniques. GB acknowledges support from the MEC and FEDER (EC) Grant SEV2014-0398, FIS2015-72245-EXP, and FPA2017-845438 and the Generalitat Valenciana under grant PROMETEOII/2017/033 and also partial support from the European Union FP10 ITN ELUSIVES (H2020MSCAITN-2015-674896) and INVISIBLES-PLUS (H2020-MSCARISE-2015-690575). This manuscript has been authored by Fermi Research Alliance, LLC under Contract no. DE-AC02-07CH11359 with the U.S. Department of Energy, Office of Science, Office of High Energy Physics. YLZ acknowledges the STFC Consolidated Grant ST/L000296/1 and the European Union's Horizon 2020 Research and Innovation Programme under Marie Skłodowska-Curie grant agreements Elusives ITN no. 674896 and InvisiblesPlus RISE no. 690575. JT and YLZ also gratefully acknowledge the hospitality of Universitat de Valencia.

Data Availability Statement This manuscript has associated data in a data repository. [Authors' comment: All data included in this manuscript are available upon request by contacting with the corresponding author.]

Open Access This article is licensed under a Creative Commons Attribution 4.0 International License, which permits use, sharing, adaptation, distribution and reproduction in any medium or format, as long as you give appropriate credit to the original author(s) and the source, provide a link to the Creative Commons licence, and indicate if changes were made. The images or other third party material in this article are included in the article's Creative Commons licence, unless indicated otherwise in a credit line to the material. If material is not included in the article's Creative Commons licence and your intended use is not permitted by statutory regulation or exceeds the permitted use, you will need to obtain permission directly from the copyright holder. To view a copy of this licence, visit http://creativecomm ons.org/licenses/by/4.0/.

Funded by $\mathrm{SCOAP}^{3}$. 


\section{Appendix A: Graviton Feynman rules}

We follow the convention of $[29,45]$ and present some of the basics of gravitational field theory in this Appendix. The full gravitational action is given by

$S_{g}=\int d^{4} x \sqrt{-g}\left(\frac{1}{4 \pi G} R+\mathcal{L}_{m}\right)$,

where $R$ is the scalar curvature and $\mathcal{L}_{m}$ is the Lagrange density for matter. For massless minimal scalar (spin-0), Dirac fermion (spin-1/2), and gauge boson (spin-1) particles, as denoted by $\phi, \psi$ and $A_{\mu}$ respectively, the $\mathcal{L}_{m}$ term are represented by

$$
\begin{aligned}
\mathcal{L}_{m}= & D_{\mu} \phi^{*} g^{\mu \nu} D_{\nu} \phi+\frac{i}{2}\left[\bar{\psi} \gamma^{a} e_{a}^{\mu} D_{\mu} \psi+\left(D_{\mu} \bar{\psi}\right) \gamma^{a} e_{a}^{\mu} \psi\right] \\
& -\frac{1}{4} g^{\mu \nu} g^{\rho \sigma} F_{\mu \rho} F_{\nu \sigma},
\end{aligned}
$$

where $F_{\mu \nu}=D_{\mu} A_{\nu}-D_{\nu} A_{\mu}$ and $D_{\mu}$ denotes the covariant derivative with respect to the gravitational field and gauge fields, and $e_{a}^{\mu}$ is the vierbein to shift frame to the local Minkowski flat frame. In the flat space background, Feynman rules for gravitational interactions are obtain by perturbing the metric

$g_{\mu \nu} \rightarrow \eta_{\mu \nu}+\kappa h_{\mu \nu}$,

where $\kappa=\sqrt{32 \pi G}$. As we work in the flat space background, the classical gravitational field is fixed at zero and $h_{\mu v}$ represents the gravitational quantum perturbation. The tree-level Feynman rules for gravitation propagator is given by

Graviton propagator : $G_{\mu \nu \rho \sigma}(p)=\frac{i \mathcal{P}_{\mu \nu \rho \sigma}}{p^{2}}$

with

$\mathcal{P}^{\mu \nu \rho \sigma}=\frac{1}{2}\left(\eta^{\mu \rho} \eta^{\nu \sigma}+\eta^{\mu \sigma} \eta^{\nu \rho}-\eta^{\mu \nu} \eta^{\rho \sigma}\right)$.

The tree-level Feynman rules for massless minimal scalar, Dirac fermion and gauge boson propagators are respectively given by

Minimal scalar propagator : $\Delta(p)=\frac{i}{p^{2}}$,

Dirac fermion propagator : $S_{F}(p)=\frac{i}{\not p}$,

Gauge boson propagator : $D_{\mu \nu}(p)=\frac{i \eta_{\mu \nu}}{p^{2}}$.

Feynman rules for interactions between graviton and fermions, minimal scalars and gauge bosons are given by $\tau_{1}, \tau_{2}$ and $\tau_{3}$ respectively.

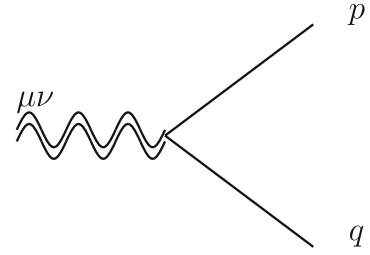

$\tau_{1}^{\mu \nu}(p, q)=\frac{i \kappa}{8}\left[(q-p)^{\mu} \gamma^{\nu}+(q-p)^{\nu} \gamma^{\mu}-2 \eta^{\mu \nu}(\not q-\not p)\right]$
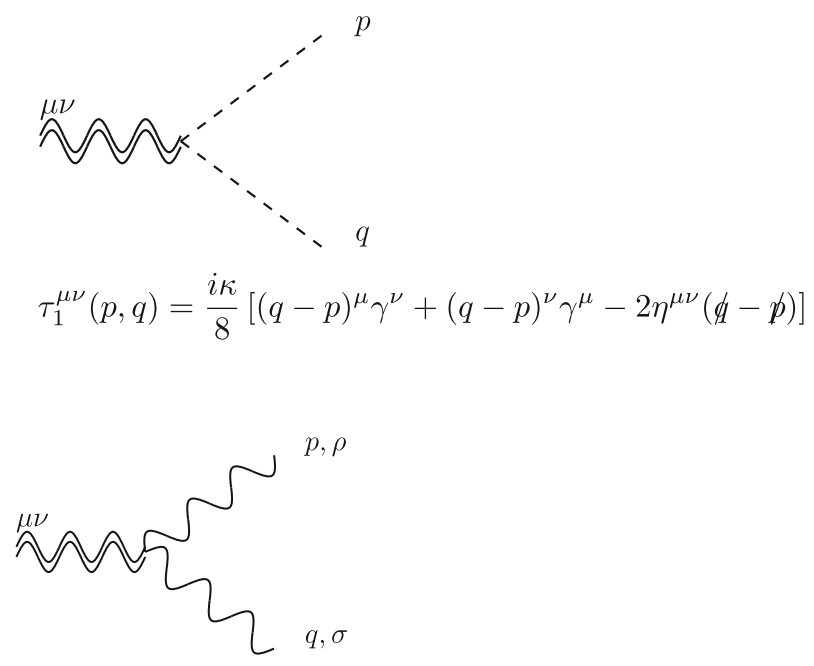

$$
\begin{gathered}
\tau_{3}^{\mu \nu \rho \sigma}(p, q)=i \kappa\left[-\mathcal{P}^{\mu \nu \rho \sigma}-\frac{1}{2} \eta^{\mu \nu} p^{\sigma} q^{\rho}+\eta^{\sigma \rho}\left(p^{\mu} q^{\nu}+p^{\nu} q^{\mu}\right)\right. \\
\left.+\frac{1}{2}\left(\eta^{\mu \sigma} p^{\nu} q^{\rho}+\eta^{\nu \sigma} p^{\rho} q^{\mu}+\eta^{\nu \rho} p^{\sigma} q^{\mu}+\eta^{\mu \rho} p^{\nu} q^{\sigma}\right)\right]
\end{gathered}
$$

where $\kappa=\sqrt{32 \pi G}$ and all momenta are assumed to be out-flowing from the vertex.

\section{Appendix B: Vacuum polarisation calculation}

In this section we show the brute force method of calculating the vacuum polarisations. Lorentz algebra was manipulated using FeynCalc [46] and the loop integration was completed using Package X [47]. The contribution from gauge bosons to the vacuum polarisation tensor is

$$
\begin{aligned}
i \Pi^{\alpha \beta, \gamma \delta}= & \int[d l] \tau_{3}^{\alpha \beta \rho \sigma}(l, q-l) \frac{-i g_{\rho \sigma}(l+q)^{2}}{(l+q)^{4}} \\
& \times \tau_{3}^{\gamma \delta \xi \omega}(-l, l-q) \frac{-i g_{\xi \omega} l^{2}}{(l)^{4}} \\
= & \kappa^{2} \int[d l] \tau_{3}^{\alpha \beta \rho \sigma}(l, q-l) \frac{g_{\rho \sigma}(l+q)^{2}}{(l+q)^{4}} \\
& \times \tau_{3}^{\gamma \delta \xi \omega}(-l, l-q) \frac{g_{\xi \omega} l^{2}}{(l)^{4}}
\end{aligned}
$$




$$
\begin{aligned}
= & \frac{2 i G}{\pi}\left[\frac{1}{30}\left(q^{\alpha} q^{\beta}-q^{2} g^{\alpha \beta}\right)\left(q^{\gamma} q^{\delta}-q^{2} g^{\gamma \delta}\right)\right. \\
& -\frac{1}{20}\left(q^{\alpha} q^{\gamma}-q^{2} g^{\alpha \gamma}\right)\left(q^{\beta} q^{\delta}-q^{2} g^{\beta \delta}\right) \\
& \left.-\frac{1}{20}\left(q^{\alpha} q^{\delta}-q^{2} g^{\alpha \delta}\right)\left(q^{\beta} q^{\gamma}-q^{2} g^{\beta \gamma}\right)\right] \\
& \times \log \left[-\frac{\mu^{2}}{q^{2}}\right] .
\end{aligned}
$$

where we have divided by a symmetry factor of two. The contribution from Dirac fermions to the vacuum polarisation tensor is

$$
\begin{aligned}
i \Pi^{\alpha \beta, \gamma \delta}= & \kappa)^{2} \int[d l] \tau_{1}^{\alpha \beta}(l, q-l) \frac{(l+q)}{(l+q)^{2}} \tau_{1}^{\gamma \delta}(-l, l-q) \frac{(l)}{(l)^{2}} \\
= & i \frac{2 G}{\pi}\left[-\frac{2}{15}\left(q^{\alpha} q^{\beta}-q^{2} g^{\alpha \beta}\right)\left(q^{\gamma} q^{\delta}-q^{2} g^{\gamma \delta}\right)\right. \\
& +\frac{1}{5}\left(q^{\alpha} q^{\gamma}-q^{2} g^{\alpha \gamma}\right)\left(q^{\beta} q^{\delta}-q^{2} g^{\beta \delta}\right) \\
& \left.+\frac{1}{5}\left(q^{\alpha} q^{\delta}-q^{2} g^{\alpha \delta}\right)\left(q^{\beta} q^{\gamma}-q^{2} g^{\beta \gamma}\right)\right] \log \left[-\frac{\mu^{2}}{q^{2}}\right] .
\end{aligned}
$$

and the contribution from minimal scalars to the vacuum polarisation tensor is

$$
\begin{aligned}
i \Pi^{\alpha \beta, \gamma \delta}= & \int[d l] \tau_{2}^{\alpha \beta}(l, q-l) \frac{i}{(l+q)^{2}} \tau_{2}^{\gamma \delta}(l+q, l) \frac{i}{(l)^{2}} \\
= & \kappa^{2} \frac{i}{16 \pi^{2}} \int[d l] \frac{\tau_{2}^{\alpha \beta}(l, q-l) \tau_{2}^{\gamma \delta}(-l, l-q)}{(l+q)^{2} l^{2}} \\
= & i \frac{2 G}{\pi}\left[\frac{1}{40}\left(q^{\alpha} q^{\beta}-q^{2} g^{\alpha \beta}\right)\left(q^{\gamma} q^{\delta}-q^{2} g^{\gamma \delta}\right)\right. \\
& +\frac{1}{240}\left(q^{\alpha} q^{\gamma}-q^{2} g^{\alpha \gamma}\right)\left(q^{\beta} q^{\delta}-q^{2} g^{\beta \delta}\right) \\
& \left.+\frac{1}{240}\left(q^{\alpha} q^{\delta}-q^{2} g^{\alpha \delta}\right)\left(q^{\beta} q^{\gamma}-q^{2} g^{\beta \gamma}\right)\right] \\
& \times \log \left[-\frac{\mu^{2}}{q^{2}}\right] .
\end{aligned}
$$

Although the graviton is not a matter field, it does have self couplings and therefore the graviton will contribute to its own vacuum polarisation. We apply the contribution from the graviton self vacuum polarisation as calculated by ' $t$ Hooft and Veltman [28],

$$
\begin{aligned}
i \Pi^{\alpha \beta, \gamma \delta}= & i \frac{2 G}{\pi}\left[\frac{23}{60}\left(q^{\alpha} q^{\beta}-q^{2} g^{\alpha \beta}\right)\left(q^{\gamma} q^{\delta}-q^{2} g^{\gamma \delta}\right)\right. \\
& -\frac{7}{40}\left(q^{\alpha} q^{\gamma}-q^{2} g^{\alpha \gamma}\right)\left(q^{\beta} q^{\delta}-q^{2} g^{\beta \delta}\right)
\end{aligned}
$$

$$
\begin{aligned}
& \left.-\frac{7}{40}\left(q^{\alpha} q^{\delta}-q^{2} g^{\alpha \delta}\right)\left(q^{\beta} q^{\gamma}-q^{2} g^{\beta \gamma}\right)\right] \\
& \times \log \left(-\frac{\mu^{2}}{q^{2}}\right) .
\end{aligned}
$$

\section{References}

1. R.N. Mohapatra, G. Senjanovic, Neutrino mass and spontaneous parity violation. Phys. Rev. Lett. 44, 912 (1980)

2. M. Gell-Mann, P. Ramond, R. Slansky, Complex spinors and unified theories. Conf. Proc. C 790927, 315-321 (1979)

3. T. Yanagida, Horizontal symmetry and masses of neutrinos. Conf. Proc. C 7902131, 95-99 (1979)

4. P. Minkowski, $\mu \rightarrow e \gamma$ at a rate of one out of $10^{9}$ muon decays? Phys. Lett. B 67, 421-428 (1977)

5. M. Magg, C. Wetterich, Neutrino mass problem and gauge hierarchy. Phys. Lett. B 94, 61-64 (1980)

6. G. Lazarides, Q. Shafi, C. Wetterich, Proton lifetime and fermion masses in an SO (10) model. Nucl. Phys. B 181, 287-300 (1981)

7. C. Wetterich, Neutrino masses and the scale of B-L violation. Nucl. Phys. B 187, 343-375 (1981)

8. R.N. Mohapatra, G. Senjanovic, Neutrino masses and mixings in gauge models with spontaneous parity violation. Phys. Rev. D 23, 165 (1981)

9. J. Schechter, J.W.F. Valle, Neutrino masses in $\mathrm{SU}(2) \mathrm{x} U(1)$ theories. Phys. Rev. D 22, 2227 (1980)

10. J. Schechter, J.W.F. Valle, Neutrino decay and spontaneous violation of lepton number. Phys. Rev. D 25, 774 (1982)

11. A. Zee, A theory of lepton number violation, neutrino majorana mass, and oscillation. Phys. Lett. B 93, 389 (1980). (Erratum: Phys. Lett. 95 B, 461 (1980))

12. T.P. Cheng, L.-F. Li, Neutrino masses, mixings and oscillations in SU(2) x U(1) models of electroweak interactions. Phys. Rev. D 22, $2860(1980)$

13. S.T. Petcov, Remarks on the zee model of neutrino mixing (mu - ? e gamma, heavy neutrino -? light neutrino gamma, etc.). Phys. Lett. B 115, 401-406 (1982)

14. K.S. Babu, Model of 'Calculable' majorana neutrino masses. Phys. Lett. B 203, 132-136 (1988)

15. N. Arkani-Hamed, S. Dimopoulos, G.R. Dvali, J. March-Russell, Neutrino masses from large extra dimensions. Phys. Rev. D 65, 024032 (2001)

16. K.R. Dienes, E. Dudas, T. Gherghetta, Neutrino oscillations without neutrino masses or heavy mass scales: a higher dimensional seesaw mechanism. Nucl. Phys. B 557, 25 (1999)

17. R.N. Mohapatra, J.W.F. Valle, Neutrino mass and baryon number nonconservation in superstring models. Phys. Rev. D 34, 1642 (1986)

18. R.N. Mohapatra, J.W.F. Valle, Neutrino mass and baryon number nonconservation in superstring models. Phys. Rev. D 34, 235 (1986)

19. S.F. King, C. Luhn, Neutrino mass and mixing with discrete symmetry. Rep. Prog. Phys. 76, 056201 (2013)

20. E.K. Akhmedov, Z.G. Berezhiani, G. Senjanovic, Planck scale physics and neutrino masses. Phys. Rev. Lett. 69, 3013-3016 (1992)

21. G. Barenboim, Gravity triggered neutrino condensates. Phys. Rev. D 82, 093014 (2010)

22. G. Barenboim, Inflation might be caused by the right: handed neutrino. JHEP 03, 102 (2009)

23. G. Dvali, L. Funcke, Small neutrino masses from gravitational theta term. Phys. Rev. D 93(11), 113002 (2016) 
24. F. Lena, R. Georg, V. Edoardo, Distinguishing dirac and majorana neutrinos by their gravi-majoron decays (2019)

25. J.F. Donoghue, General relativity as an effective field theory: the leading quantum corrections. Phys. Rev. D 50, 3874-3888 (1994)

26. Y. Nambu, G. Jona-Lasinio, Dynamical model of elementary particles based on an analogy with superconductivity. 1. Phys. Rev. 122, 345-358 (1961)

27. C.D. Roberts, A.G. Williams, Dyson-Schwinger equations and their application to hadronic physics. Prog. Part. Nucl. Phys. 33, 477-575 (1994)

28. G. Hooft, M.J.G. Veltman, One loop divergencies in the theory of gravitation. Ann. Inst. H. Poincare Phys. Theor. A20, 69-94 (1974)

29. B.-B. Niels, E. Jannik, Quantum gravity, effective fields and string theory. Ph.D. thesis, Bohr Inst. (2004)

30. C.T. Hill, P.A.N. Machado, A.E. Thomsen, J. Turner, Scalar democracy. Phys. Rev. D 100(1), 015015 (2019)

31. S. Abel, E. Mølgaard, F. Sannino, Complete asymptotically safe embedding of the standard model. Phys. Rev. D 99(3), 035030 (2019)

32. G. Dvali, I. Sawicki, A. Vikman, Dark matter via many copies of the standard model. JCAP 0908, 009 (2009)

33. F.T. Hawes, A.G. Williams, Chiral symmetry breaking in quenched massive strong coupling QED in four-dimensions. Phys. Rev. D 51, 3081-3089 (1995)

34. F.T. Hawes, A.G. Williams, C.D. Roberts, Renormalization and chiral symmetry breaking in quenched QED in arbitrary covariant gauge. Phys. Rev. D 54, 5361-5372 (1996)

35. J. Bardeen, L.N. Cooper, J.R. Schrieffer, Theory of superconductivity. Phys. Rev. 108, 1175-1204 (1957)

36. M. Aker et al., An improved upper limit on the neutrino mass from a direct kinematic method by KATRIN (2019)
37. O. Abe, Y. Hoshino, Stability of fermion and momentum cutoff in quantum gravity in flat background space-time. Phys. Lett. 139B, 167-169 (1984)

38. O. Abe, Chiral symmetry breaking in quantum gravity in flat background space-time. Prog. Theor. Phys. 73, 1560 (1985)

39. N. Arkani-Hamed, S. Dimopoulos, G.R. Dvali, The hierarchy problem and new dimensions at a millimeter. Phys. Lett. B 429, 263-272 (1998)

40. L. Randall, R. Sundrum, A large mass hierarchy from a small extra dimension. Phys. Rev. Lett. 83, 3370-3373 (1999)

41. C. Csáki, S. Lombardo, O. Telem, TASI Lectures on nonsupersymmetric BSM Models, in Proceedings Theoretical Advanced Study Institute in Elementary Particle Physics: Anticipating the Next Discoveries in Particle Physics (TASI 2016), Boulder, CO, USA, June 6-July 1, 2016. (WSP, WSP, 2018), p. 501-570

42. X. Calmet, The lightest of black holes. Mod. Phys. Lett. A 29(38), 1450204 (2014)

43. X. Calmet, R. Casadio, The horizon of the lightest black hole. Eur. Phys. J. C 75(9), 445 (2015)

44. L.S. Christiane, F. Lena, L. Matthias, C. Erminia, Reconstruction of the neutrino mass as a function of redshift (2021)

45. J.F. Donoghue, M.M. Ivanov, A. Shkerin, EPFL lectures on general relativity as a quantum field theory (2017)

46. V. Shtabovenko, R. Mertig, F. Orellana, New developments in FeynCalc 9.0. Comput. Phys. Commun. 207, 432-444 (2016)

47. H.H. Patel, Package-X: a mathematica package for the analytic calculation of one-loop integrals. Comput. Phys. Commun. 197, 276-290 (2015) 